\title{
Evaluation Method of Jamming Effect on ISAR Based on Target Recognition Characters
}

\author{
Cui Rui Pan jifei Han yinyong Sheng aiguo \\ Electronic Engineering Institute \\ Hefei, China \\ cuirui2002@sohu.com
}

\begin{abstract}
Some methods used to evaluate the jamming effect of common radar are unsuitable to the inverse synthetic aperture radar (ISAR). By comparing the change of target recognition characters, a novel evaluation method of jamming effect on ISAR is presented in the paper. Two typical noise barrage jamming modes, amplitude modulation (AM) noise jamming and frequency modulation (FM) noise jamming are carried out in the paper, the results of simulation prove the method is corrective and effective.
\end{abstract}

Keywords-ISAR; barrage jamming; target recognition characters; evaluation;

\section{INTRODUCTION}

The Inverse Synthetic Aperture Radar is a high resolution imaging radar, since it has the ability to reconstruct a two dimension high-resolution image of non-cooperative moving target, such as space objects, air targets and ships; it has been widely used for target classification, recognition and identification in military field $^{[1]-[2]}$. As a main part of Electronic Countermeasures (ECM), Evaluation method of jamming effect on ISAR attracts much more attention.

The image is the final product of ISAR and target recognition is its final purpose. The recognition probability more depends on the choice of target characters, which are the base of target recognition. By comparing the change of target recognition characters, a novel evaluation method of jamming effect on ISAR based on the target recognition characters is presented in the paper, which can reflect the impact on the target recognition by the jamming.

\section{THE DESCRIPTION OF TARGET RECOGNITION CHARACTERS}

The target characters are the base of target classification, the target recognition correct probability more depends on the choice of characters. The characters which used for recognize plan should have the invariant character of rotating, but the image with same shape and different size could represent different type plans. Multi-characters should be used to get higher recognition probability. The characters which used for target recognition based on the ISAR image should have the invariant characters of shifting, rotation and scaling, such as the character of moment and the character of shape. In the paper, we choose the character of moment and area to evaluate the jamming effect on ISAR.

A. Character of Moment

One two-dimension grey-degree picture $f(x, y), x=0, \ldots . ., M-1, y=0, \ldots ., N-1$ is given, and then the geometry origin Moment with $p+q$ rank can be determined as following formula ${ }^{[3]}$ :

$$
m_{p q}=\sum_{x=0}^{M-1 N-1} \sum_{y=0}^{p} y^{q} f(x, y), p, q=0,1,2, \ldots \ldots
$$

In order to make these instantaneous variables fixed when the target is moving, the center Moment can be determined:

$$
\mu_{p q}=\sum_{x=0}^{M-1 N-1}(x-\bar{x})^{p}(y-\bar{y})^{q} f(x, y), p, q=0,1,2, \ldots .
$$

Where $\bar{x}=\frac{m_{10}}{m_{00}}, \bar{y}=\frac{m_{01}}{m_{00}}$

In order to make center Moment unrelated to zoom, it can be changed as following:

$$
\begin{gathered}
\eta_{p q}=\frac{\mu_{p q}}{\mu_{00}^{\gamma}} \\
\gamma=\frac{p+q}{2}+1 \quad p+q=2334 \ldots . .
\end{gathered}
$$

Then, the following four moment invariants are invariable with shifting, rotation and scaling:

$$
\begin{gathered}
m_{1}=\eta_{20}+\eta_{02} \\
m_{2}=\left(\eta_{20}-\eta_{02}\right)^{2}+4 \eta_{11}^{2} \\
m_{3}=\left(\eta_{30}-3 \eta_{12}\right)^{2}+\left(3 \eta_{21}-\eta_{03}\right)^{2} \\
m_{4}=\left(\eta_{30}+\eta_{12}\right)^{2}+\left(\eta_{21}+\eta_{03}\right)^{2}
\end{gathered}
$$

So, the moment feature vector $m=\left[\begin{array}{llll}m_{1} & m_{2} & m_{3} & m_{4}\end{array}\right]$ can be used for target recognition based on the ISAR image.

\section{B Character of Area}

One two-dimension grey-degree picture $f(x, y), x=0, \ldots . ., M-1, y=0, \ldots ., N-1$ is given, 
and then the character of area can be determined as following formula:

$$
A=\sum_{x=0}^{M-1} \sum_{y=0}^{N-1} f(x, y)
$$

\section{THE SIMULATION OF JAMMING EFFECT ON ISAR}

The ideal plan point target model is adopted to carry out the simulation of barrage jamming on ISAR in the paper. The parameters of system are represented as follows: Signal carrier frequency $\mathrm{f} 0=10 \mathrm{GHz}$, bandwidth $\mathrm{B}=200 \mathrm{MHz}, \quad$ Pulse-width $\tau=2 u s \quad, \mathrm{PRF}=200 \mathrm{HZ}$, the radial velocity of plan $\mathrm{v}=15 \mathrm{~m} / \mathrm{s}$. Noise AM jamming and noise FM jamming are carried out in the paper. The signal of noise AM can be described as ${ }^{[4]}$ :

$$
J(t)=\left(U_{0}+u_{n}(t)\right) \cos \left(2 \pi f_{0} t+\varphi\right)
$$

Where modulated noise $u_{n}(t)$ is a wide-sense stationary stochastic processes, its mean is zero and variance is $\sigma_{n}^{2}$, amplitude of carrier wave is $U_{0}$, carrier frequency is $f_{0}$, original phase of carrier wave is $\varphi$.The signal of noise FM can be expressed as:

$$
J(t)=U_{0} \cos \left[2 \pi f_{j} t+2 \pi k_{F M} \int_{0}^{t} u(\tau) d \tau+\phi\right]
$$

Where modulated noise $u(t)$ is a wide-sense stationary stochastic processes, its mean is zero and variance is $\sigma_{n}^{2}$, amplitude of carrier wave is $U_{0}$, carrier frequency is $f_{i}$, original phase of carrier wave is $\varphi$, coefficient of frequency modulation is $k_{F M}$.

Fig. 1 and Fig. 2 are the image that have been jammed in the two jamming mode with the same JSR (8dB and $15 \mathrm{~dB}$ ), when JSR is $8 \mathrm{~dB}$, from Fig.2a, we can still distinguish the plan even though it has been jammed, however the image in Fig.3a has been destroyed badly. When JSR is $15 \mathrm{~dB}$, both of them have been destroyed badly, but we still can see clearly that the jamming effect of noise FM is better than the effect of noise AM.

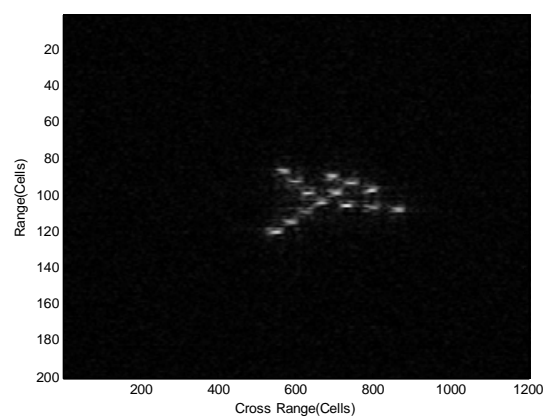

a. $\mathrm{JSR}=10 \mathrm{~dB}$

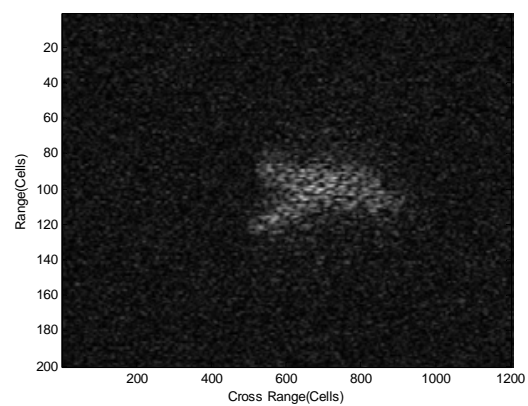

b. JSR $=16 \mathrm{~dB}$

Fig.1 ISAR image with noise AM jamming

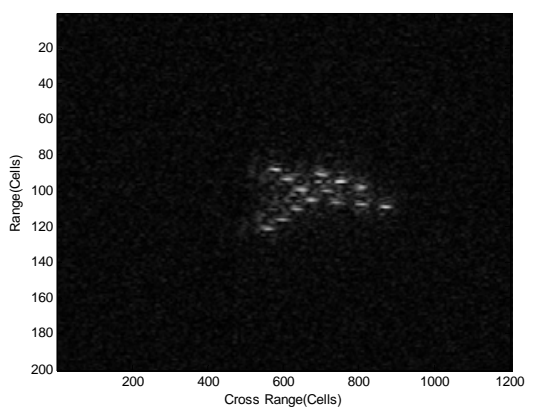

a. $J S R=10 \mathrm{~dB}$

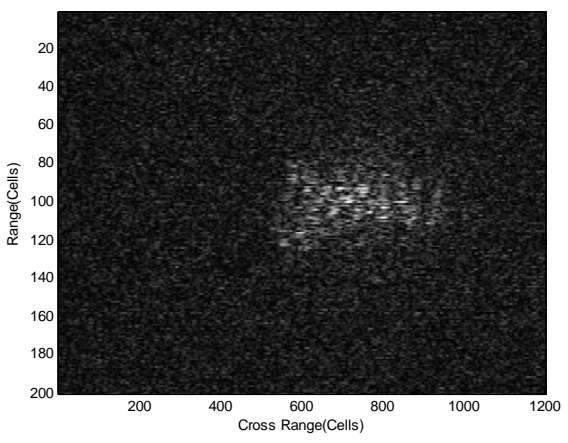

b. JSR $=16 \mathrm{~dB}$

Fig.2 ISAR image with noise FM jamming

\section{QUANTITATIVE JAMMING EFFECT EVALUATION}

These characters keep unchanged when the target is expanded or moved or rotated, so they are always used to recognize the target. They are changed because the target image is destroyed by noise. As a result, the recognition probabilities fall. From the point of view, the evaluation method of jamming effect on ISAR based on target recognition characters is proposed in the paper.

The moment of target original image is $\phi_{0}$, the area of target original image is $S_{0}$, then the target character vector of ISAR is built: $x=\left(\phi_{0}, S_{0}\right)$, the target character vector of ISAR that been jammed is also built: $y=\left(\phi_{1}, S_{1}\right)$,the distance of them can be determined as 
follows $^{[5]}$ :

$$
d(x, y)=\sqrt{\left(\phi_{1}-\phi_{0}\right)^{2}+\left(S_{1}-S_{0}\right)^{2}}
$$

Considering the difference of importance degree of two characters, formula (11) can be overwritten:

$$
d(x, y)=\sqrt{k\left(\phi 1-\phi_{0}\right)^{2}+(1-k)\left(S_{1}-S_{0}\right)^{2}}
$$

$k$ is the positive real number, which represent the importance degree of two characters.

With the same JSR, if the relationship between jamming mode $\mathrm{A}$ and jamming model $\mathrm{B}$ meet the formula $d_{A, J S R 0}(x, y)>d_{B, J S R 0}(x, y)$, then we can consider the jamming effect of mode $A$ is better than mode $B$.

According toFig1 and Fig2; the distance of two images can be calculated according to (12), the relationship between JSR and the distance of characters can be shown as Fig.3:

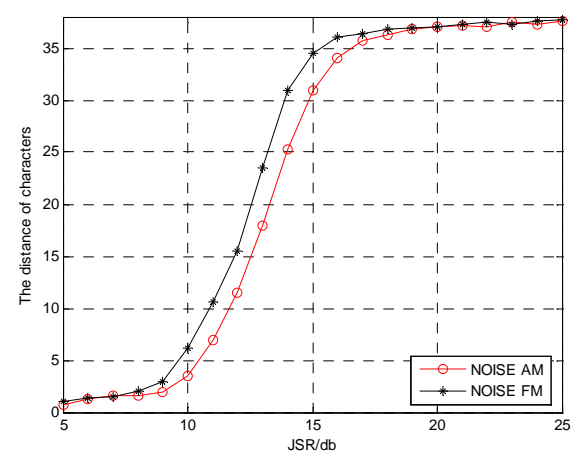

Fig. 3 curve of the distance of characters

The conclusions can be got from the figure 3 :

1 . The curve of the distance of characters rise clearly, it proves that the target have been jammed effectively by two jamming mode, and that with the increase of JSR, the jamming effect become better and better.

2 . With the same JSR, the distance of characters that jammed by noise FM is bigger than that jammed by noise AM. It proves that the jamming effect of noise FM is better than noise AM, which is consistent with the conclusion that got from the Fig 1 and Fig 2.

3. Two curves get together when JSR is bigger than $17 \mathrm{~dB}$, which mean the image have been jammed badly and the noise have made evaluation criterion invalidated.

\section{CONCLUSIONS}

The military application of ISAR become more and more important, it has a very big significance to study the jamming and jamming effect evaluation technology on ISAR. The target recognition characters are analyzed deeply and applied to evaluate the jamming effect on ISAR. Through the change of the distance of characters, the method of quantitative jamming effect evaluation on ISAR is carried out. It has such merit as computed easily, also has been proved corrective and effective by the results of simulation.

\section{REFERENCES}

[1] CHEN C C. Target-Motion-Induced Radar Imaging [J]. IEEE Trans on AES.1980, 16(1):2-14.

[2] Bao zheng, Xing Mengdao, WangTong, Technology of radar imaging, Publishing house of Electronic Industry,2005, pp.6-9.

[3] Dudani, BA, Breeding, KJ and Mc Ghee, RG . Aircraft Indentification by Moment Invariants. IEEE.Trans, Comput, 1977, $26: 39-46$

[4] LENG Chuan-hang, FU YU-Sheng, PI Yi-Ming, HOU Yin-ming. Research on simulation of interference on inverse synthetic aperture radar[J]. the technology of electronic warfare, 2005, (5) : 35-39.

[5] Cui Rui, Xue Lei, Wang Bo. Evaluation method of jamming effect on ISAR Based on Equivalent number of looks[J]. Systems Engineering and Electronics.2008,30(5):887-888. 